\title{
WNT11 is a direct target of early growth response protein 1
}

\author{
JuHwan Kim ${ }^{1}$, Euitaek Jung ${ }^{1}$, Sung Shin Ahn ${ }^{1}$, Hyunjin Yeo ${ }^{1}$, Jeong Yeon Lee ${ }^{1}$, Jeong Kon Seo ${ }^{2}$, Young Han Lee ${ }^{1,3} \mathcal{E}$ \\ Soon Young Shin ${ }^{1,3, *}$ \\ ${ }^{1}$ Department of Biological Sciences, Sanghuh College of Lifesciences, Konkuk University, Seoul 05029, ${ }^{2}$ Central Research Facilities, Ulsan \\ National Institute of Science and Technology, Ulsan 44919, ${ }^{3}$ Cancer and Metabolism Institute, Konkuk University, Seoul 05029, Korea
}

\begin{abstract}
WNT11 is a member of the non-canonical Wnt family and plays a crucial role in tumor progression. However, the regulatory mechanisms underlying WNT11 expression are unclear. Tumor necrosis factor-alpha ( $\mathrm{TNF} \alpha$ ) is a major inflammatory cytokine produced in the tumor microenvironment and contributes to processes associated with tumor progression, such as tumor invasion and metastasis. By using site-directed mutagenesis and introducing a serial deletion in the 5'-regulatory region of WNT11, we observed that TNF $\alpha$ activates the early growth response 1 (EGR1)-binding sequence (EBS) in the proximal region of WNT11 and that the transcription factor EGR1 is necessary for the TNF $\alpha$-induced transcription of WNT11. EGR1 bound directly to the EBSs within the proximal 5'-regulatory region of WNT11 and ectopic expression of EGR1 stimulated WNT11 promoter activity, whereas the knockdown of EGR1 expression by RNA interference reduced TNF $\alpha$-induced WNT11 expression in T47D breast cancer cells. We also observed that mitogen-activated protein kinases (MAPK), extracellular signalregulated kinase (ERK), c-Jun N-terminal kinase (JNK), and p38 kinase mediated TNF $\alpha$-induced transcription of WNT11 via EGR1. Our results suggest that EGR1 directly targets WNT11 in response to TNF $\alpha$ stimulation in breast cancer cells. [BMB Reports 2020; 53(12): 628-633]
\end{abstract}

\section{INTRODUCTION}

Wnt signaling pathways represent a group of highly evolutionarily conserved signal transduction pathways that control various physiological responses, including embryonic body axis formation, cell fate specification, cell proliferation, and cell migration (1). To date, at least $19 \mathrm{Wnt}$ proteins have been

*Corresponding author. Tel: +82-2-2030-7946; Fax: +82-2-3437-9781; E-mail: shinsy@konkuk.ac.kr

https://doi.org/10.5483/BMBRep.2020.53.12.052

Received 9 March 2020, Revised 22 April 2020, Accepted 6 July 2020

Keywords: 5'-regulatory region, Early growth response 1, Mitogenactivated protein kinase, Tumor necrosis factor $\alpha$, Wnt family member 11 identified in humans. All Wnt proteins bind and activate the Frizzled receptors, which transduce biological signals to the appropriate downstream targets (2). Wnt signaling is characterized by the $\beta$-catenin-dependent canonical pathway and the $\beta$-catenin-independent non-canonical pathway (3). Emerging evidence has implicated canonical and non-canonical Wnt signaling pathways in the development of human cancers, including gastrointestinal cancers, hepatocellular carcinoma, leukemia, melanoma, and breast cancers $(4,5)$.

WNT11 plays a crucial role in several morphological processes associated with embryogenesis, including anterior-posterior axis elongation through a $\beta$-catenin-dependent canonical or -independent non-canonical pathway (6). WNT11 promotes the proliferation and transformation of intestinal epithelial cells (7) and the downregulation of its receptor, frizzled-7, apart from reducing the survival, invasion, and metastasis of colon cancer cells (8). Consistent with the above, WNT11 overexpression promotes the proliferation and migration of several tumor cells, including cervical, breast, prostate, and colon cancer cells $(9,10)$. These results suggest that WNT11 signaling plays a crucial role in cell proliferation, invasion, and metastasis during carcinogenesis (11).

The transcription of WNT11 is controlled by multiple factors, including lymphoid enhancer-binding factor 1 (LEF1), GATA transcription factor (12), and E26 transforming sequence (ETS)related gene (ERG), an ETS family transcription factor involved in hematopoiesis (13). In breast cancer cells, Chromatin immunoprecipitation-linked target site cloning was used to characterize WNT11 as an estrogen receptor target gene (14).

Tumor necrosis factor-alpha (TNF $\alpha$ ) is a major inflammatory cytokine produced by tumor cells, tumor-associated fibroblasts, and infiltrated inflammatory cells in the tumor microenvironment (15). TNF $\alpha$ stimulates various inflammatory cytokines and chemokines and plays a crucial role in processes associated with tumor progression, including tumor invasion and metastasis (15-18). However, the role of TNF $\alpha$ in the regulation of WNT11 expression remains largely elusive. Owing to the role of TNF $\alpha$ in tumor progression, we hypothesized that TNF $\alpha$ may control WNT11 transcription. To evaluate this possibility, we isolated the $5^{\prime}$-regulatory region of the WNT11 gene and evaluated its expression levels in response to TNF $\alpha$ stimulation in the T47D breast cancer cell line. We observed that TNF $\alpha$ stimulates WNT11 transcription by activating the

ISSN: 1976-670X (electronic edition)

Copyright (C) 2020 by the The Korean Society for Biochemistry and Molecular Biology

(c) This is an open-access article distributed under the terms of the Creative Commons Attribution Non-Commercial License (http://creativecommons.org/licenses/by-nc/4.0) which permits unrestricted non-commercial use, distribution, and reproduction in any medium, provided the original work is properly cited. 
EGR1-binding cis-acting element within the 5'-regulatory region of WNT11. We also observed that the three major mitogen-activated protein kinases (MAPKs), extracellular signalregulated kinase (ERK), c-Jun N-terminal kinase (JNK), and p38 kinase, mediated TNF $\alpha$-induced WNT11 expression via EGR1 upregulation.

\section{RESULTS AND DISCUSSION}

\section{TNF $\alpha$ upregulates WNT11 expression in T47D breast cancer cells}

We first examined the basal mRNA expression levels of WNT11 in various breast and colon cancer cells. MCF-7 and T47D breast cancer cells expressed relatively high levels of WNT11 mRNA, whereas no expression or low levels of expression were observed in non-transformed MCF10A breast epithelial cells and MDA-MB-231 breast cancer cells (Fig. 1A, left panels). Among the different colon cancer cells, the basal levels of WNT11 mRNA were relatively high in SW620 and HT29 cells, while the same was not observed in SW480 cells (Fig. 1A, right panels). We selected and characterized T47D

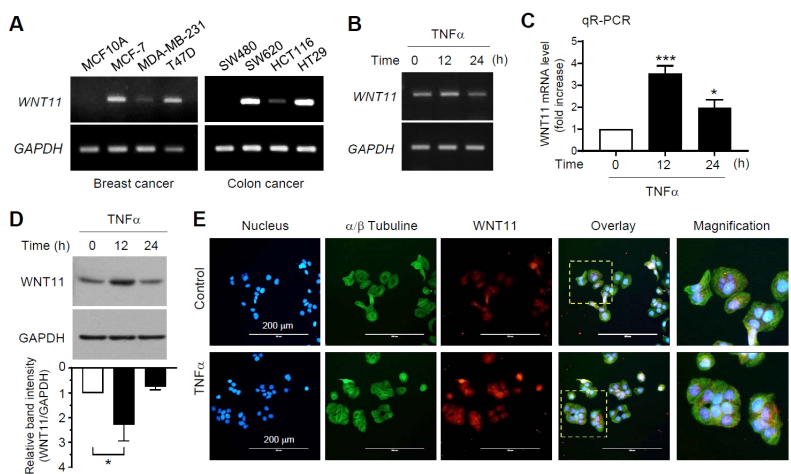

Fig. 1. Effect of TNF $\alpha$ on WNT11 expression. (A) Basal expression of WNT11 mRNA in various cancer cells. (B, C) T47D cells were treated with $10 \mathrm{ng} / \mathrm{ml}$ TNF $\alpha$ for 12 and $24 \mathrm{~h}$. Total RNA was isolated, and the levels of WNT11 mRNA were determined using RTPCR (B) and quantitative real-time PCR (qR-PCR) (C). The levels of GAPDH mRNA were determined as an internal control. In $(C)$, the 0 -time mRNA level was set to 1 after adjustment to the GAPDH levels. Data are presented as mean + S.D. $(n=3)$. ${ }^{* P}=0.0131$; $* * * \mathrm{P}<0.001$ by Dunnett's multiple comparisons test. (D) WNT11 levels were measured by immunoblotting. GAPDH levels were determined as an internal control. Band intensities were measured using the ImageJ software. After adjustment to the GAPDH levels, the 0-time band intensity was set to 1. Data are presented as mean + S.D. $(n=3)$. *P $=0.0127(n=3)$ by Dunnett's multiple comparisons test. (E) T47D cells were treated with PBS (control) or $10 \mathrm{ng} / \mathrm{ml} \mathrm{TNF} \alpha$, and then incubated with antibodies against $\alpha / \beta$ tubulin or WNT11 for $2 \mathrm{~h}$, followed by incubation with Alexa Fluor 488-conjugated (for $\alpha / \beta$-tubulin; green signal) and Alexa Fluor 555-conjugated (for WNT11; red signal) secondary antibodies for $30 \mathrm{~min}$. Nuclear DNA was stained with $1 \mu \mathrm{g} / \mathrm{ml}$ Hoechst 33258 for $10 \mathrm{~min}$ (blue staining). White bar size, $50 \mu \mathrm{m}$. Arrows, WNT11stained spots. cells exhibiting moderate WNT11 expression levels to investigate WNT11 mRNA expression following TNF $\alpha$ stimulation. An increase in the mRNA levels of WNT11 was detected within $12 \mathrm{~h}$, followed by a reduction at $24 \mathrm{~h}$ after TNF $\alpha$ stimulation, whereas the mRNA levels of the glyceraldehyde 3-phosphate dehydrogenase gene $(G A P D H)$ remained unaltered, as revealed by reverse transcription (RT)-PCR (Fig. 1B) and quantitative real-time PCR (qR-PCR) (Fig. 1C). Similar results were obtained in MDA-MB-231 breast cancer cells (Supplementary Fig. S1A) and HCT116 colon cancer cells (Supplementary Fig. S2B). TNF $\alpha$-induced elevation of the WNT11 protein levels was confirmed by immunoblotting in T47D cells (Fig. 1D) and MDA-MB-231 cells (Supplementary Fig. S1B). Immunofluorescence studies also showed WNT11 antibodystaining after TNF $\alpha$ stimulation; however, no staining was observed in the PBS-treated control (Fig. 1E). These data suggest that WNT11 expression can be regulated by TNF $\alpha$ in T47D breast cancer cells.

TNF $\alpha$ stimulates $W N T 11$ promoter activity by activating the EGR1-binding cis-acting element in the proximal promoter region of the 5 '-regulatory region

To investigate whether TNF $\alpha$ stimulates WNT11 transcription, a fragment of the 5'-regulatory region spanning nucleotides -997 to +74 was isolated, and the effect exerted by TNF $\alpha$ on the activation of the WNT11 promoter was assessed. We observed that TNF $\alpha$ significantly enhanced the promoter-reporter activity (Fig. 2A), suggesting that TNF $\alpha$ enhances WNT11 expression at the transcriptional level. To delineate the promoter region that is responsible for TNF $\alpha$-induced WNT11 promoter activation, we designed a series of deletion constructs and mapped the TNF $\alpha$ response region. Upon TNF $\alpha$ stimulation, the shortest reporter construct $(-53 /+74)$ continued to exhibit induction (Fig. 2A), suggesting that the TNF $\alpha$-inducible region of the WNT11 promoter is located between nucleotides -53 and +74 .

To identify the functional cis-acting element responsible for TNF $\alpha$-induced WNT11 gene transcription, we analyzed the transcription factor regulatory sequences between nucleotides -53 and +74 using a web-based Matlnspector transcription factor search tool (http://www.genomatix.de/). We found two putative early growth response protein 1 (EGR1)-binding sequences (EBSs) spanning nucleotides -22 to +18 (named EBS-1, -22 to -4 , and EBS-2, -1 to +18 ) (Fig. 2B). EGR1, also known as zinc finger protein 225 (Zif268) or nerve growth factor-induced clone A (NGFI-A), is a $\mathrm{Cys}_{2} \mathrm{His}_{2}$-type zinc finger protein that exhibits Fos-like induction kinetics in response to various mitogenic stimuli and DNA damage signals (19). The target genes of EGR1 are associated with multiple physiological responses, including cell proliferation, differentiation, apoptosis, and inflammation, in a variety of cell types $(19,20)$. However, the role of EGR1 in WNT11 transcription remains elusive.

To assess the functional role of these putative EBSs in 


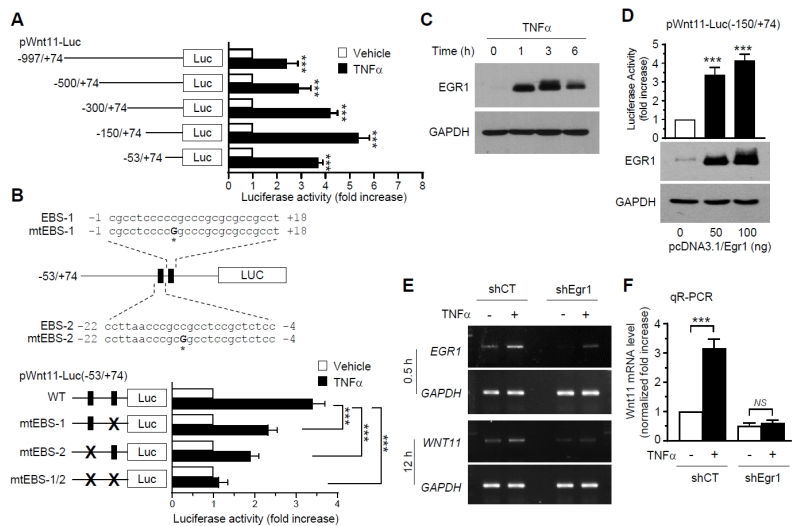

Fig. 2. Role of EGR1 in TNF $\alpha$-induced WNT11 expression. (A) HEK293 cells were transiently transfected with $0.2 \mu \mathrm{g}$ of a series of 5 '-deletion constructs. After treating with vehicle (PBS) or $10 \mathrm{ng} /$ $\mathrm{ml} T \mathrm{TNF} \alpha$, luciferase activities were measured. (B) Sequence elements of EBS between -22 to +18 in the $-53 /+73$ construct (top subfigure). HEK293 cells were transiently transfected with site-specific mutants derived from the $-53 /+74$ construct. After treatment with the vehicle (PBS) or with $10 \mathrm{ng} / \mathrm{ml} \mathrm{TNF} \alpha$, luciferase activities were measured. EBS, EGR1-binding sequence; mtEBS, mutation of EBS. Asterisk $(*)$ indicates a mutation site. Luciferase activity data are presented as mean + S.D. $(n=3)$. ${ }^{* * * P}<0.001$ by Sidak's multiple comparisons test. (C) T47D cells were treated with $10 \mathrm{ng} / \mathrm{ml}$ TNF $\alpha$ for 0,3 , and $6 \mathrm{~h}$. After preparing the nucleus-enriched fractions, EGR1 levels were measured by immunoblotting. (D) HEK293 cells were transiently cotransfected with $0.2 \mu \mathrm{g}$ of the $-150 /+74$ construct and EGR1 expression plasmid (pcDNA3.1/Egr1). Forty-eight hours later, the cells were harvested, and luciferase activities were measured. Data are presented as mean + S.D. $(n=3)$. The transfected EGR1 levels were confirmed by immunoblotting. (E and F) T47D transfectants stably expressing control scrambled shRNA (shCT) or EGR1 shRNA (shEgr1) were treated with $10 \mathrm{ng} / \mathrm{ml}$ TNF $\alpha$ for 0.5 or $12 \mathrm{~h}$. Expression levels of the EGR1 and WNT11 mRNAs were measured by RT-PCR (E), and WNT11 mRNA levels were quantitated by quantitative real-time PCR (qR-PCR) (F). qR-PCR data are presented as mean + S.D. $(n=3)$. NS, not significant; ***P $<0.001$ by Sidak's multiple comparisons test.

TNF $\alpha$-induced WNT11 transcription, we introduced site-directed mutations into the EBSs. Damage to the EBS-1 (mtEBS-1) or EBS-2 (mtEBS-2) motifs in the pWnt11-Luc $(-53 /+74)$ significantly reduced TNF $\alpha$-induced reporter activity compared to that in the wild-type (WT) construct (Fig. 2B, bottom graph). Damage to both EBS-1 and EBS-2 sites resulted in an almost complete loss of TNF $\alpha$ inducibility.

$\mathrm{TNF} \alpha$ induces EGR1 expression in various cell types (21-24). Consistently, we observed that EGR1 expression increased in a time-dependent manner upon TNF- $\alpha$ stimulation (Fig. 2C). To determine whether EGR1 transactivates WNT11, we transfected the $-150 /+74$ construct into T47D cells, along with an expression plasmid for EGR1. Exogenous overexpression of EGR1 enhanced the WNT11 promoter-reporter activity in a plasmid concentration-dependent manner (Fig. 2D). These results suggest that EGR1 can transactivate WNT11 in T47D cells.

To further investigate the role of EGR1 in TNFo-induced

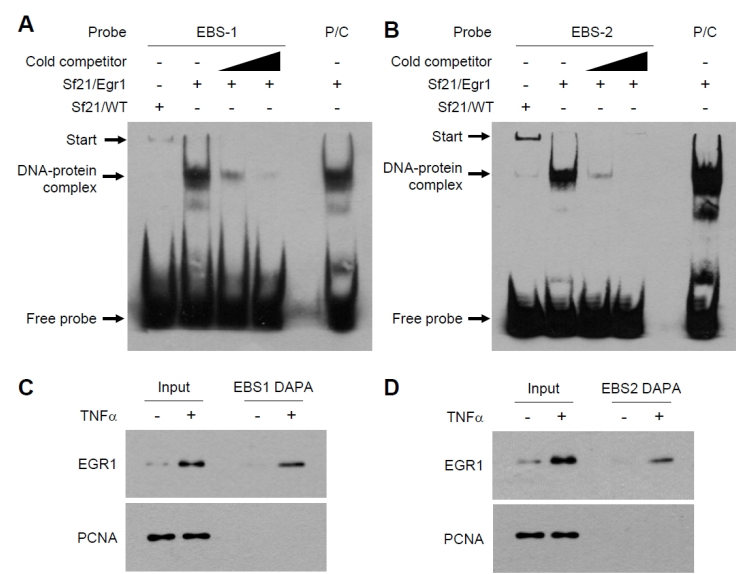

Fig. 3. Binding of EGR1 to putative EBS motifs of WNT11. (A, B) EBS-1 (A) and EBS-2 (B) probes were incubated with lysates of Sf21 cells expressing EGR1 (Sf21/EGR1) or with the wild-type (Sf21/ WT) in the presence or absence of an unlabeled probe (cold competitor). Samples were separated by non-denaturing $6 \%$ acrylamide gel electrophoresis and visualized using streptavidin-conjugated horseradish peroxidase. (C and D) T47D cells were treated with $10 \mathrm{ng} / \mathrm{ml}$ TNF $\alpha$ for $1 \mathrm{~h}$ or were left untreated. Nuclear extracts were prepared and incubated with biotinylated EBS-1 (C) or EBS-2 (D), followed by the addition of streptavidin-conjugated agarose beads. After $1 \mathrm{~h}$, the agarose beads were washed and boiled for 5 min with $2 \times$ Laemmli sample buffer. Immunoblotting was performed using antiEGR1 or anti-PCNA antibodies. Input, an aliquot of the nuclear extract; DAPA, DNA-affinity precipitation assay.

WNT11 transcription, we established T47D transfectants expressing lentiviral shRNA against EGR1 (T47D/shEgr1) and a scrambled control (T47D/shCT). The stable knockdown of basal and TNFo-induced expression of EGR1 mRNA was confirmed by RT-PCR after TNF $\alpha$ treatment (Fig. 2E, top panels). The ability of TNF $\alpha$ to induce the mRNA expression of WNT11 was substantially attenuated when EGR 1 expression in T47D/shEgr1 was reduced compared to that in T47D/shCT cells (Fig. 2E, bottom panels). qR-PCR analysis revealed that TNF $\alpha$ elevated WNT11 mRNA expression by $3.17 \pm 0.306-$ fold in T47D/shCT cells, while it reduced WNT11 mRNA expression by $0.600 \pm 0.100$-fold in T47D/shEgr 1 cells (Fig. $2 \mathrm{~F})$. These results suggest that the EBS motifs in the promoter region are necessary for the TNF $\alpha$-induced transcription of WNT11.

\section{EGR1 binds to the EBS motif and transactivates WNT11 transcription}

To determine whether the EBS motif can serve as a binding site for EGR1, we performed the non-radioactive electrophoretic mobility shift assay (EMSA) using Sf21 insect cell lysates overexpressing EGR1 (Sf21/Egr1) and biotinylated EBS oligonucleotide probes. Biotinylated consensus EBS oligonucleotides were used as a positive control. We observed that the biotinylated EBS-1 (Fig. 3A) and EBS-2 (Fig. 3B) probes formed 
DNA-protein complexes. These complexes competed with a molar excess of an unlabeled probe. These data suggest that EGR1 binds specifically to the EBS motifs within the proximal WNT11 promoter region.

To further corroborate the binding of EGR1 to the EBS motifs in WNT11, we adopted an alternative approach using the DNA-affinity precipitation assay (DAPA). Biotinylated EBS-1 and EBS-2 oligonucleotides were incubated with nuclear extracts from T47D cells. Upon TNF $\alpha$ stimulation, the levels of EGR1 and nuclear protein PCNA increased in the whole nuclear extract (input) (Fig. 3C and D; bottom panels). After pull-down with streptavidin-agarose beads, oligonucleotide-binding proteins were eluted and immunoblotted with an anti-EGR1 or anti-PCNA antibody. We observed that while EBS-1 and EBS-2 bound EGR1 in response to TNF $\alpha$ stimulation, they did not bind PCNA (Fig. 3C and D; top panels). These data suggest that the proximal EBS sites located between nucleotides -22 and +18 in the WNT11 promoter region bind EGR1; however, they do not exhibit any off-target effect.

\section{MAPKs mediate TNF $\alpha$-induced WNT11 expression via EGR1 upregulation}

Transcription factor ETS like-1 protein (ELK1) and serum response factor (SRF) complex transactivate the serum response element (SRE) in the 5'-regulatory region of the EGR1 gene. MAPK pathways control EGR1 expression via phosphorylation of ELK-1 in response to TNF $\alpha$ stimulation in several cell types $(21,25,26)$. To determine which MAPK pathways are responsible for EGR1-mediated WNT11 expression, we validated the active status of TNF $\alpha$-induced MAPKs in serumstarved T47D cells. The phosphorylation levels of ERK1/2 at Thr-202/Tyr-204, JNK1/2 at Thr-183/Tyr-185, and p38 kinase at Thr-180/Tyr-182 increased within $30 \mathrm{~min}$ in response to TNF $\alpha$ stimulation (Fig. 4A). Pretreatment with MAPK inhibitors, including the MAPK kinase (MEK) inhibitor U0126, JNK inhibitor SP600125, and p38 kinase inhibitor SB203580, substantially abrogated the TNF $\alpha$-induced accumulation of EGR1 proteins (Fig. 4B). Consistent with EGR1 expression results, all MAPK inhibitors significantly reduced TNF $\alpha$-induced WNT11 expression; in particular, U0126 and SP600125 showed a strong inhibitory effect compared to SB203580 (Fig. $4 C$ ). These data suggest that all three major MAPK pathways contribute to EGR1-mediated WNT11 expression in response to TNF $\alpha$ exposure in T47D breast cancer cells.

The current study identified the role of EGR1 in TNF $\alpha$ induced WNT11 expression in T47D human breast cancer cells. In response to TNF $\alpha$ stimulation, EGR1 directly bound to the EBS in the WNT11 promoter region and stimulated WNT11 transcription. All three major MAPKs, ERK, JNK, and p38 kinases, were involved in TNFo-induced EGR1 upregulation. We conclude that WNT11 is a direct target of the EGR1 in response to TNF $\alpha$ stimulation, further expending our understanding of the regulatory mechanism of WNT11 expression in the tumor microenvironment. As WNT11 activates
A
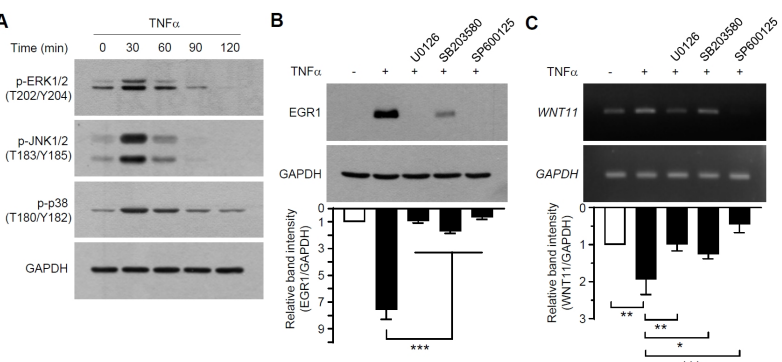

Fig. 4. Role of MAPKs in TNF $\alpha$-induced WNT11 expression. (A) T47D cells were treated with $10 \mathrm{ng} / \mathrm{ml}$ TNF $\alpha$ for various durations (0-1200 $\mathrm{min}$ ). Immunoblotting was performed using a phospho-specific antibody against ERK1/2 (Thr202/Tyr204), JNK1/2 (Thr183/ Tyr185), or p38 kinase (Thr180/Tyr182). GAPDH levels were determined as an internal control. (B, C) T47D cells were pretreated with U0126 $(10 \mu \mathrm{M})$, SB203580 $(20 \mu \mathrm{M})$, or SP600125 $(20 \mu \mathrm{M})$ for $30 \mathrm{~min}$ and then stimulated with $10 \mathrm{ng} / \mathrm{ml} \mathrm{TNF} \alpha$. After 1 and $12 \mathrm{~h}$, the levels of EGR1 (B) and WNT11 mRNAs (C) were measured by immunoblotting and RT-PCR, respectively. The band intensities were measured relative to the GAPDH levels using the ImageJ software. Bars represent the mean \pm S.D. $(n=3)$. ${ }^{*} \mathrm{P}=$ $0.0149 ;{ }^{*} * \mathrm{P}=0.0017 ; * * * \mathrm{P}<0.001$ by Dunnett's multiple comparison test.

cancer cell motility and metastasis (27) and reduction of WNT11 expression decreases the ability of breast cancer cells to migrate (9), EGR1-regulated WNT11 expression can be a promising therapeutic target for suppressing metastasis of breast cancer. Further in vivo studies are warranted to verify the clinical relevance of targeting the TNF $\alpha$-EGR1-WNT11 axis to prevent tumor metastasis in breast cancer.

\section{MATERIALS AND METHODS}

\section{Cell and reagents}

Detailed cells and reagents used in this study were described in Supplementary materials.

\section{Reverse transcription-PCR (RT-PCR)}

Total RNA was isolated using a TRIzol RNA extraction kit (Invitrogen). First-strand cDNA was synthesized using an iScript CDNA synthesis kit (Bio-Rad, Hercules, CA, USA). The PCR conditions were as follows: hold for $5 \mathrm{~min}$ at $94^{\circ} \mathrm{C}$, followed by 30 cycles of denaturation at $94^{\circ} \mathrm{C}(30 \mathrm{~s})$, annealing at $55^{\circ} \mathrm{C}$ (30 s), and elongation at $72^{\circ} \mathrm{C}(1 \mathrm{~min})$. The gene-specific PCR primers were as follows: WNT11 forward, 5' GAT CCC AAG CCA ATA AAC TGA TGC GTC T-3'; WNT11 reverse, 5'-GTC TTG TTG CAC TGC CTG TCT TGT GTC C-3'; GAPDH forward, 5' CCAAGGAGTAAGAAACCCTGGAC-3'; GAPDH reverse, 5'-GGGCCGAGT TGGGATAGG G-3'. The amplified products were electrophoresed in $1 \%$ agarose gel.

\section{Immunoblotting}

Immunoblot analysis was performed as described previously 
(21). Detailed experimental methods were described in Supplementary materials.

\section{Immunofluorescence}

T47D cells cultured on coverslips were either treated with the vehicle (PBS) or $10 \mathrm{ng} / \mathrm{ml}$ TNF $\alpha$ for $18 \mathrm{~h}$, followed by fixation, permeabilization, and incubation with primary antibodies against WNT11 and $\alpha / \beta$-tubulin (for counterstaining). After 2 $h$, the cells were incubated for 30 min with secondary antibodies conjugated with Alexa Fluor 555 (for $\alpha / \beta$-tubulin; red signal) and Alexa Fluor 488 (for WNT11, green signal). Nuclear DNA was stained using $1 \mu \mathrm{g} / \mathrm{ml}$ Hoechst 33258 for an additional $10 \mathrm{~min}$ (blue signal). Fluorescently-stained cells were examined under an EVOS FL fluorescence microscope (Advanced Microscopy Group, Bothell, WA, USA).

\section{Construction and mutagenesis of human WNT11 promoter-reporter constructs}

Detailed experimental methods for the generation of the human WNT11 promoter-reporter constructs and primer sequences were described in Supplementary materials.

\section{WNT11 promoter-reporter assay}

HEK293T cells cultured in 12-well plates were transfected with $0.2 \mu \mathrm{g}$ WNT11 promoter constructs using Lipofectamine 2000 (Invitrogen), according to the manufacturer's instructions. Detailed experimental methods for promoter-reporter assay were described in Supplementary materials.

\section{Electrophoretic mobility shift assay (EMSA)}

EMSA was performed using a LightShift Chemiluminescence EMSA kit, according to the manufacturer's instructions (ThermoFisher Scientific, Waltham, MA, USA). Biotin-labeled deoxyoligonucleotide probes specific for EBS-1 (5'-CCT TAA CCC GCC GCC TCC GCT CTC C-biotin-3'), EBS-2 (5'-AGG CGG CGC GCG GGC GGG GGA GGC G-biotin-3'), and the consensus EBS sequence (5'-GGA TCC AGC GGG GGC GAG CGG GGG CGA-biotin-3') were obtained from Macrogen. For the competition assay, 1 and 2.5 pmole of the unlabeled EBS-1 and EBS-2 probes (competitor) were added, respectively. DNAprotein complexes were electrophoresed in non-denaturing $6 \%$ polyacrylamide gels and visualized using an $\mathrm{ECL}$ chemiluminescence system (GE Healthcare Life Science).

\section{DNA-affinity precipitation assay (DAPA)}

DAPA was performed as reported previously (28). Briefly, nuclear extracts $(35 \mu \mathrm{g})$ were incubated with $500 \mathrm{pmol}$ of biotinylated EBS-1 (5'-biotin-CCT TAA CCC GCC GCC TCC GCT CTC C-3') or EBS-2 (5'-biotin-AGG CGG CGC GCG GGC GGG GGA GGC G-3') oligonucleotide and streptavidin-conjugated agarose beads (Invitrogen) for $1 \mathrm{~h}$, as described previously (29). After washing thrice with PBS, the pellets were boiled with $2 \times$ Laemmli sample buffer and immunoblot analysis was performed using antibodies against EGR1 or PCNA (off-target control).

\section{EGR1 silencing using RNA interference}

T47D cells were incubated with lentiviral shRNA (TRCN 0000273850; MISSION ${ }^{\mathbb{R}}$ shRNA; Sigma-Aldrich) targeting EGR $\overline{1}$, according to the manufacturer's instructions. After $2 \mathrm{wk}$, the silencing of EGR1 expression was determined by immunoblotting.

\section{Statistical analysis}

Statistical significance of results was determined using one-way analysis of variance (ANOVA), followed by Sidak's multiple comparisons test or Dunnett's multiple comparisons test using the GraphPad Prism version 8.3.1 software (GraphPad Software Inc., La Jolla, CA, USA). A P-value $<0.05$ was considered statistically significant.

\section{ACKNOWLEDGEMENTS}

This study was supported by the Basic Science Research Program through the National Research Foundation of Korea (NRF), funded by the Ministry of Science, ICT and Future Planning (Grant no. 2020R1A2C1005845), Republic of Korea. This paper was supported by the KU Research Professor Program of Konkuk University and by the Konkuk University Researcher Fund in 2019.

\section{CONFLICTS OF INTEREST}

The authors have no conflicting interests.

\section{REFERENCES}

1. Goldsberry WN, Londono A, Randall TD, Norian LA and Arend RC (2019) A review of the role of wnt in cancer immunomodulation. Cancers (Basel) 11, 771

2. Nusse R and Clevers H (2017) Wnt/beta-catenin signaling, disease, and emerging therapeutic modalities. Cell 169, 985-999

3. van Andel $H$, Kocemba KA, Spaargaren $M$ and Pals ST (2019) Aberrant Wnt signaling in multiple myeloma: molecular mechanisms and targeting options. Leukemia 33, 1063-1075

4. Zhan T, Rindtorff N and Boutros M (2017) Wnt signaling in cancer. Oncogene 36, 1461-1473

5. Shi J, Zheng $\mathrm{H}$ and Yuan L (2019) High NDRG3 expression facilitates HCC metastasis by promoting nuclear translocation of beta-catenin. BMB Rep 52, 451-456

6. Tao Q, Yokota C, Puck H et al (2005) Maternal wnt11 activates the canonical wnt signaling pathway required for axis formation in Xenopus embryos. Cell 120, 857-871

7. Ouko L, Ziegler TR, Gu LH, Eisenberg LM and Yang VW (2004) Wnt11 signaling promotes proliferation, transformation, and migration of IEC6 intestinal epithelial cells. J Biol Chem 279, 26707-26715

8. Ueno K, Hazama S, Mitomori S et al (2009) Down-regu- 
lation of frizzled-7 expression decreases survival, invasion and metastatic capabilities of colon cancer cells. $\mathrm{Br} \mathrm{J}$ Cancer 101, 1374-1381

9. Dwyer MA, Joseph JD, Wade HE et al (2010) WNT11 expression is induced by estrogen-related receptor alpha and beta-catenin and acts in an autocrine manner to increase cancer cell migration. Cancer Res 70, 9298-9308

10. Wei $\mathrm{H}$, Wang $\mathrm{N}$, Zhang $\mathrm{Y}$, Wang $\mathrm{S}$, Pang $X$ and Zhang $\mathrm{S}$ (2016) Wnt-11 overexpression promoting the invasion of cervical cancer cells. Tumour Biol 37, 11789-11798

11. Uysal-Onganer P and Kypta RM (2012) Wnt11 in 2011 the regulation and function of a non-canonical Wnt. Acta Physiol (Oxf) 204, 52-64

12. Katoh $M$ and Katoh $M$ (2009) Integrative genomic analyses of WNT11: transcriptional mechanisms based on canonical WNT signals and GATA transcription factors signaling. Int J Mol Med 24, 247-251

13. Mochmann LH, Bock J, Ortiz-Tanchez J et al (2011) Genome-wide screen reveals WNT11, a non-canonical WNT gene, as a direct target of ETS transcription factor ERG. Oncogene 30, 2044-2056

14. Lin Z, Reierstad S, Huang CC and Bulun SE (2007) Novel estrogen receptor-alpha binding sites and estradiol target genes identified by chromatin immunoprecipitation cloning in breast cancer. Cancer Res 67, 5017-5024

15. Ham B, Fernandez MC, D'Costa Z and Brodt P (2016) The diverse roles of the TNF axis in cancer progression and metastasis. Trends Cancer Res 11, 1-27

16. Balkwill $F$ (2009) Tumour necrosis factor and cancer. Nat Rev Cancer 9, 361-371

17. Coussens LM and Werb Z (2002) Inflammation and cancer. Nature 420, 860-867

18. Joyce JA and Pollard JW (2009) Microenvironmental regulation of metastasis. Nat Rev Cancer 9, 239-252

19. Gashler A and Sukhatme VP (1995) Early growth response protein 1 (Egr-1): prototype of a zinc-finger family of transcription factors. Prog Nucleic Acid Res Mol Biol 50, 191224

20. Milbrandt J (1987) A nerve growth factor-induced gene encodes a possible transcriptional regulatory factor. Science
238, 797-799

21. Son SW, Min BW, Lim Y, Lee YH and Shin SY (2008) Regulatory mechanism of TNFalpha autoregulation in $\mathrm{HaCaT}$ cells: the role of the transcription factor EGR-1. Biochem Biophys Res Commun 374, 777-782

22. Cao XM, Guy GR, Sukhatme VP and Tan YH (1992) Regulation of the Egr-1 gene by tumor necrosis factor and interferons in primary human fibroblasts. J Biol Chem 267, 1345-1349

23. Grimbacher B, Aicher WK, Peter $\mathrm{HH}$ and Eibel $\mathrm{H}$ (1998) TNF-alpha induces the transcription factor Egr-1, pro-inflammatory cytokines and cell proliferation in human skin fibroblasts and synovial lining cells. Rheumatol Int 17, 185-192

24. Chaudhary LR, Cheng SL and Avioli LV (1996) Induction of early growth response- 1 gene by interleukin- 1 beta and tumor necrosis factor-alpha in normal human bone marrow stromal an osteoblastic cells: regulation by a protein kinase C inhibitor. Mol Cell Biochem 156, 69-77

25. Utreras E, Futatsugi A, Rudrabhatla P et al (2009) Tumor necrosis factor-alpha regulates cyclin-dependent kinase 5 activity during pain signaling through transcriptional activation of p35. J Biol Chem 284, 2275-2284

26. Mishra JP, Mishra S, Gee K and Kumar A (2005) Differential involvement of calmodulin-dependent protein kinase II-activated AP-1 and c-Jun N-terminal kinase-activated EGR-1 signaling pathways in tumor necrosis factoralpha and lipopolysaccharide-induced CD44 expression in human monocytic cells. J Biol Chem 280, 26825-26837

27. Luga V, Zhang L, Viloria-Petit AM et al (2012) Exosomes mediate stromal mobilization of autocrine Wnt-PCP signaling in breast cancer cell migration. Cell 151, 15421556

28. Wu KK (2006) Analysis of protein-DNA binding by streptavidin-agarose pulldown. Methods Mol Biol 338, 281-290

29. Kim J, Jung E, Choi J, Min DY, Lee YH and Shin SY (2019) Leptin is a direct transcriptional target of EGR1 in human breast cancer cells. Mol Biol Rep 46, 317-324 